\title{
Analysis of General Input State Dependent Working Vacation Queue with Changeover Time
}

\author{
Vijaya Laxmi Pikkala and Suchitra Vepada \\ Department of Applied Mathematics, Andhra University, Visakhapatnam 530003, India \\ Correspondence should be addressed to Vijaya Laxmi Pikkala; vijaya_iit2003@yahoo.co.in
}

Received 7 February 2014; Accepted 20 March 2014; Published 23 April 2014

Academic Editors: G. Ciobanu and F. W. S. Lima

Copyright ( 2014 V. L. Pikkala and S. Vepada. This is an open access article distributed under the Creative Commons Attribution License, which permits unrestricted use, distribution, and reproduction in any medium, provided the original work is properly cited.

\begin{abstract}
We consider a finite buffer GI/M(n)/1 queue with multiple working vacations and changeover time, where the server can keep on working but at a slower speed during the vacation period. Moreover, the amount of service demanded by a customer is conditioned by the queue length at the moment service is begun for that customer. We provide a recursive algorithm using the supplementary variable technique to numerically compute the stationary queue length distribution of the system. Finally, some numerical results of the model are presented to show the parameter effect on various performance measures.
\end{abstract}

\section{Introduction}

Queueing systems with vacations are considered to be effective tools in modeling and analyzing complex computer and communication networks and several other engineering systems where the server can utilize the idle time for different purposes; see Doshi [1] and Tian and Zhang [2]. On the other hand, numerous situations exist where the server remains active during the vacation period. Servi and Finn [3] introduced a class of semivacation policies for the analysis of an $M / M / 1$ queue. In this the server renders service to the queue with a lower service rate, known as working vacation (WV). In multiple working vacations (MWV) policy when a vacation ends and the system is not empty, a service period begins with the normal service rate; otherwise, the server takes another vacation.

Baba [4] extended Servi and Finn's [3] work to GI/M/1/ MWV queue using the matrix-analytic approach and derived the expressions of distributions for queue length at an arrival epoch and the steady state distribution for the waiting time. Banik et al. [5] discussed the GI/M/1/N queue with MWV and obtained some important performance measures. Zhang and Hou [6] generalized GI $/ M / 1 / N$ queue with a variant of MWV. They employed the supplementary variable and embedded Markov chain methods to obtain the queue length distribution at different epochs.
At a service completion epoch during regular service, the server remains in the system for some time instead of leaving for a WV immediately. This time is known as changeover time during which if a customer arrives, the server starts service with regular service rate; otherwise, it leaves for a WV. Li and Zhu [7] provided an explicit formula for the Laplace transform of the additional delay for $M / G / 1$ queues with delayed vacations and exhaustive service discipline. Dong and Doo [8] investigated the $G / M / 1$ queue with changeover time and multiple vacations. They derived the joint distribution of the queue length and the remaining service (or vacation) time at arbitrary time by using the supplementary variable method and calculated the virtual waiting time distribution. The steady state behavior of an $M / M^{(a, b, d)} / 1$ queueing system with multiple vacations and delayed vacations has been investigated by Jain and Singh [9]. Recently, Vijaya Laxmi and Seleshi [10] extended this study to a renewal input $\mathrm{GI} / M^{(a, c, b)} / 1$ queue with $\mathrm{WV}$ and changeover times.

Many standard queueing systems operate on the assumption that input and service parameters are dependent on the state of the system, which reduces the waiting time of customers. For example, in telecommunication systems at the packet switch (router) when its buffer size increases, a controller drops the arriving packets with an increasing 
probability. In human based service systems, it is known that there is a strong correlation between the volume of work demanded from a human and his/her productivity. A computational algorithm of GI/M(n)/1/K queue with state dependent vacations and $N$-policy with multiple vacations are analyzed by Chao and Rahman [11]. Using the supplementary variable technique, a recursive algorithm has been explained by Goswami et al. [12] for obtaining the system length distributions at prearrival and arbitrary epochs for a finite buffer state dependent $\mathrm{GI} / M / 1$ queue with MWV.

The above literature survey indicates that so far no work has been focused on state dependent MWV queue with changeover time, which is applicable in production, manufacturing, traffic signal and telecommunication systems, and so forth. For example, in a packet switched network, the router is an interconnection device that attaches two or more networks. It takes charge of receiving packets and forwarding them to the next hop, according to some routing information in its routing table. If the routes are available in the routing table, router will serve the packets depending on the number of packets which is termed as state dependent routing. This procedure routes the packets to the least loaded disks. This offers significant throughput benefits over state independent routing. To collect the complete routing information, the router may exchange its routing information with the other routers. When there is no packet to be sent, a "sleep mode" is operated for the purpose of power saving. If a packet arrives during this sleep mode period, the router returns to the awakening state immediately and it transmits packets. Meanwhile, under the maintenance, the router can serve the packet at slower speed which can reduce waiting times and economize the cost. In queueing terminology, the router, routing at slower speed, state dependent routing, and sleep mode correspond to the server, working vacation, state dependent services, and changeover times, respectively.

Motivated by such situations of sleep mode operations, which conserve energy and further reduce the waiting times, this paper aims to focus on finite buffer state dependent queue with MWV and changeover time. We assumed the service times during service period, vacation period, and vacation times are exponentially distributed. We provide a recursive method using the supplementary variable technique and treating the remaining interarrival time as the supplementary variable, to develop the steady state system length distributions at prearrival and arbitrary epochs. A computational algorithm is presented to compute the stationary system length distribution. Some performance measures and numerical results have been illustrated in the form of tables and graphs.

The paper is structured as follows. Next section presents the description of the model. Steady state equations and relation between prearrival and arbitrary epochs are derived in Section 3. A computational algorithm is presented in Section 4 followed by various performance measures in Section 5. Section 5 also contains some numerical results to show the effectiveness of the model parameters and conclusions are given in Section 6.

\section{Description of the Model}

Let us consider a GI/M(n)/1/N/MWV queue with changeover time where $N$ is the capacity of the system. We assume that the interarrival times of successive arrivals are independent and identically distributed (i.i.d.) random variables with cumulative distribution function $A(x)$, probability density function $a(x), x \geq 0$, Laplace-Stiltjes (LS) transform $A^{*}(\theta)$, and mean interarrival time $1 / \lambda=-A^{*(1)}(0)$, where $h^{(1)}(0)$ denotes the first derivative of $h(\theta)$ evaluated at $\theta=0$. The service rate during a regular busy period is assumed to be an exponentially distributed random variable. When there are $n$ customers present in the system before beginning a service, the server serves them with a rate $\mu_{n}, 1 \leq n \leq N$. The server takes WV whenever the system becomes empty. When a vacation ends and if there are customers in the queue, a regular busy period begins and server serves the queue with its usual service rate; otherwise, the server takes another vacation. The vacation rate and service rate during any $\mathrm{WV}$ period are also assumed to be exponentially distributed random variables with $\gamma_{n}, \eta_{n}(1 \leq n \leq N)$, respectively, where there are $n$ customers present in the system during a vacation. Whenever the queue becomes empty, instead of leaving for a WV immediately the server will remain in the system for a certain period of time, called changeover time and which is assumed to be exponentially distributed with parameter $\varsigma$. Let $\mu(\eta)$ and $\gamma$ be the mean service rate during regular busy period (WV) and mean vacation rate, respectively, and they are given by $\mu=\sum_{n=1}^{N} \mu_{n} / N, \eta=\sum_{n=1}^{N} \eta_{n} / N, \gamma=\sum_{n=1}^{N} \gamma_{n} / N$. The customers are served by a single server on first-come first-served (FCFS) discipline. The arrival times, service time, and changeover times are mutually independent.

Let us define the state of the server as

$$
\xi(t)= \begin{cases}0, & \text { if the server is in WV period, } \\ 1, & \text { if the server is in regular busy } \\ \text { period or in changeover time. }\end{cases}
$$

We define the joint probability densities of system length $N_{s}(t)$, state of the server $\xi(t)$, and the remaining interarrival time for the customer $U(t)$, respectively, as

$$
\begin{aligned}
& P_{n, j}(u) d u \\
& =\lim _{t \rightarrow \infty} P\left\{N_{s}(t)=n, u \leq U(t) \leq u+d u, \xi(t)=j\right\}, \\
& j=0,1, \quad 0 \leq n \leq N, \quad u \geq 0 .
\end{aligned}
$$

The above probabilities at steady state are denoted by $P_{n, j}(u)$. In particular, $P_{0,1}(0)$ denotes the changeover time probability.

\section{Steady State Equations and Solution}

In order to obtain the queue length distributions at arbitrary epochs, we first develop the differential-difference equations 
using the supplementary variable technique and they are written as

$$
\begin{gathered}
-P_{0,0}^{(1)}(x)=\varsigma P_{0,1}(x)+\eta_{1} P_{1,0}(x), \\
-P_{n, 0}^{(1)}(x)=-\delta_{n} P_{n, 0}(x)+\eta_{n+1} P_{n+1,0}(x) \\
+a(x) P_{n-1,0}(0), \quad 1 \leq n \leq N-1, \\
-P_{N, 0}^{(1)}(x)=-\delta_{N} P_{N, 0}(x)+a(x)\left(P_{N-1,0}(0)+P_{N, 0}(0)\right) \\
-P_{0,1}^{(1)}(x)=-\varsigma P_{0,1}(x)+\mu_{1} P_{1,1}(x), \\
-P_{n, 1}^{(1)}(x)=-\mu_{n} P_{n, 1}(x)+\mu_{n+1} P_{n+1,1}(x) \\
+\gamma_{n} P_{n, 0}(x)+a(x) P_{n-1,1}(0), \quad 1 \leq n \leq N-1, \\
-P_{N, 1}^{(1)}(x)=-\mu_{N} P_{N, 1}(x)+\gamma_{N} P_{N, 0}(x) \\
+a(x)\left(P_{N-1,1}(0)+P_{N, 1}(0)\right),
\end{gathered}
$$

where $\delta_{n}=\gamma_{n}+\eta_{n}, P_{n, j}(0), j=0,1,0 \leq n \leq N$ are the respective rates of arrivals; that is, an arrival is about to occur. Let us define the Laplace transforms of $P_{n, j}(x)$ as $P_{n, j}^{*}(\theta)=$ $\int_{0}^{\infty} e^{-\theta x} P_{n, j}(x) d x, \operatorname{Re} \theta \geq 0$. Hence, $P_{n, j} \equiv P_{n, j}^{*}(0)$ are the joint probabilities where there are $n$ customers in the system and the server is in state $j, j=0,1$. Multiplying (3) to (8) by $e^{-\theta x}$ and integrating with respect to $x$ from 0 to $\infty$ yield

$$
\begin{aligned}
& -\theta P_{0,0}^{*}(\theta)=\varsigma P_{0,1}^{*}(\theta)+\eta_{1} P_{1,0}^{*}(\theta)-P_{0,0}(0), \\
& \left(\delta_{n}-\theta\right) P_{n, 0}^{*}(\theta)=\eta_{n+1} P_{n+1,0}^{*}(\theta) \\
& +A^{*}(\theta) P_{n-1,0}(0)-P_{n, 0}(0), \\
& 1 \leq n \leq N-1 \text {, } \\
& \left(\delta_{N}-\theta\right) P_{N, 0}^{*}(\theta)=A^{*}(\theta)\left(P_{N-1,0}(0)+P_{N, 0}(0)\right)-P_{N, 0}(0) \text {, } \\
& (\varsigma-\theta) P_{0,1}^{*}(\theta)=\mu_{1} P_{1,1}^{*}(\theta)-P_{0,1}(0), \\
& \left(\mu_{n}-\theta\right) P_{n, 1}^{*}(\theta)=\mu_{n+1} P_{n+1,1}^{*}(\theta)+\gamma_{n} P_{n, 0}^{*}(\theta) \\
& +A^{*}(\theta) P_{n-1,1}(0)-P_{n, 1}(0), \\
& 1 \leq n \leq N-1 \\
& \left(\mu_{N}-\theta\right) P_{N, 1}^{*}(\theta) \\
& =\gamma_{N} P_{N, 0}^{*}(\theta) \\
& +A^{*}(\theta)\left(P_{N, 1}(0)+P_{N-1,1}(0)\right)-P_{N, 1}(0) \text {. }
\end{aligned}
$$

Further, adding (9) to (14) and taking $\theta \rightarrow 0$, we obtain the following result:

$$
\sum_{n=0}^{N} P_{n, 0}(0)+\sum_{n=0}^{N} P_{n, 1}(0)=\lambda .
$$

The left-hand side denotes the mean number of entrances into the system per unit time and is equal to mean arrival rate $\lambda$.

Substituting $\theta=\delta_{n}$ in (11) and (10), we get

$$
\begin{gathered}
P_{N-1,0}(0)=\frac{1-A^{*}\left(\delta_{N}\right)}{A^{*}\left(\delta_{N}\right)} P_{N, 0}(0), \quad n=N, \\
P_{n-1,0}(0)=\frac{P_{n, 0}(0)-\eta_{n+1} P_{n+1,0}^{*}\left(\delta_{n}\right)}{A^{*}\left(\delta_{n}\right)}, \quad n=N-1, \ldots, 1,
\end{gathered}
$$

where $P_{n, 0}^{*}(\theta)$ are given from (11) and (10) as

$$
\begin{gathered}
P_{N, 0}^{*}(\theta)=\frac{A^{*}(\theta)-A^{*}\left(\delta_{N}\right)}{\left(\delta_{N}-\theta\right)} P_{N, 0}(0), \quad n=N, \\
P_{n, 0}^{*}(\theta)=\frac{\eta_{n+1} P_{n+1,0}^{*}(\theta)+A^{*}(\theta) P_{n-1,0}(0)-P_{n, 0}(0)}{\left(\delta_{n}-\theta\right)}, \\
n=N-1, \ldots, 1 .
\end{gathered}
$$

Substituting $\theta=\mu_{n}$ in (14) and (13), we get

$$
\begin{gathered}
P_{N-1,1}(0)=\frac{1-A^{*}\left(\mu_{N}\right)}{A^{*}\left(\mu_{N}\right)} P_{N, 1}(0)-\frac{\gamma_{N}}{A^{*}\left(\mu_{N}\right)} P_{N, 0}(0), \\
n=N, \\
P_{n-1,1}(0)=\frac{P_{n, 1}(0)-\mu_{n+1} P_{n+1,1}^{*}\left(\mu_{n}\right)-\gamma_{n} P_{n, 0}^{*}\left(\mu_{n}\right)}{A^{*}\left(\mu_{n}\right)}, \\
n=N-1, \ldots, 1,
\end{gathered}
$$

where $P_{n, 1}^{*}(\theta)$ are given by the following:

$$
\begin{array}{r}
P_{N, 1}^{*}(\theta) \\
=\frac{\gamma_{N} P_{N, 0}^{*}(\theta)+A^{*}(\theta)\left(P_{N-1,1}(0)+P_{N, 1}(0)\right)-P_{N, 1}(0)}{\left(\mu_{N}-\theta\right)}, \\
n=N,
\end{array}
$$

$$
\begin{aligned}
& P_{n, 1}^{*}(\theta) \\
& =\frac{\gamma_{n} P_{n, 0}^{*}(\theta)+\mu_{n+1} P_{n+1,1}^{*}(\theta)+A^{*}(\theta) P_{n-1,1}(0)-P_{n, 1}(0)}{\left(\mu_{n}-\theta\right)},
\end{aligned}
$$$$
n=N-1, \ldots, 1 \text {. }
$$

For $\theta=\delta_{n}, P_{n, 0}^{*}(\theta)$ are given by

$$
\begin{array}{r}
P_{N, 0}^{*}(\theta)=-\left[A^{*(1)}(\theta)\left(P_{N-1,0}(0)+P_{N, 0}(0)\right)\right], \\
P_{n, 0}^{*}(\theta)=-\left[\eta_{n+1} P_{n+1,0}^{*(1)}(\theta)+A^{*(1)}(\theta) P_{n-1,0}(0)\right], \\
1 \leq n \leq N-1 .
\end{array}
$$


For $\theta=\mu_{n}, P_{n, 1}^{*}(\theta)$ are given by

$$
P_{N, 1}^{*}(\theta)=-\left[\gamma_{N} P_{N, 0}^{*(1)}(\theta)+A^{*(1)}(\theta)\left(P_{N-1,1}(0)+\pi_{N, 1}(0)\right)\right]
$$

$$
\begin{array}{r}
P_{n, 1}^{*}(\theta) \\
=-\left[\gamma_{n} P_{n, 0}^{*(1)}(\theta)+\mu_{n+1} P_{n+1,0}^{*(1)}(\theta)+A^{*(1)}(\theta) P_{n-1,1}(0)\right], \\
1 \leq n \leq N-1 .
\end{array}
$$

It can be seen from the above set of expressions that we can easily evaluate $P_{n, j}(0), j=0,1,0 \leq n \leq N$.

3.1. Relation between Steady State Distributions at Arbitrary and Prearrival Epochs. To obtain the arbitrary epoch probabilities, we develop the relations between number of customers in the system at prearrival and arbitrary epochs as follows. Let $P_{n, j}^{-}, j=0,1,(0 \leq n \leq N)$ represent the probability of $n$ customers in the system at prearrival epoch when the server is in state $j$. Applying Bayes theorem, for $j=0,1$, we have

$$
P_{n, j}^{-}=\frac{P_{n, j}(0)}{\lambda}, \quad j=0,1,0 \leq n \leq N .
$$

Now, to obtain the steady state probabilities at arbitrary epochs, we develop a relation between prearrival and arbitrary epoch probabilities. Setting $\theta=0$ in (11), (10), and (14)(12) and using (28), we obtain

$$
P_{N, 0}=\frac{\lambda}{\delta_{N}} P_{N-1,0}^{-}
$$

$$
\begin{gathered}
P_{n, 0} \\
=\frac{\lambda}{\delta_{n}}\left[P_{n-1,0}^{-}-\frac{\gamma_{n+1}}{\delta_{n+1}} P_{n, 0}^{-}-\sum_{j=n+1}^{N-1} \frac{\gamma_{j+1}}{\delta_{j+1}} \times \prod_{k=n+1}^{j} \frac{\eta_{k}}{\delta_{k}} P_{j, 0}^{-}\right], \\
1 \leq n \leq N-1, \\
P_{n, 1}=\frac{\lambda}{\mu_{n}}\left[\begin{array}{l}
P_{n-1,1}^{-}+\frac{\lambda}{\mu_{N}}\left[\frac{\gamma_{N}}{\delta_{N}} P_{N-1,0}^{-}+P_{N-1,1}^{-}\right] \\
\left.\delta_{n-1,0}^{-}+\sum_{j=n}^{N-1} \frac{\gamma_{j+1}}{\delta_{j+1}} \times \prod_{k=n}^{j} \frac{\eta_{k}}{\delta_{k}} P_{j, 0}^{-}\right] \\
P_{0,1}=\frac{\lambda}{\varsigma}\left[\frac{\gamma_{1}}{\delta_{1}} P_{0,0}^{-}+\sum_{j=n}^{N-1} \frac{\gamma_{j+1}}{\delta_{j+1}} \times \prod_{k=n}^{j} \frac{\eta_{k}}{\delta_{k}} P_{j, 0}^{-}\right] \\
P_{0,0}=1-\sum_{i=1}^{N} P_{n, 0}-\sum_{i=0}^{N} P_{n, 1} \cdot
\end{array}\right.
\end{gathered}
$$

Remark 1. When $\varsigma \rightarrow \infty$, that is, there is no changeover time, the model reduces to GI/M(n)/1/N queue with MWV and our results match numerically with Goswami et al. [12].

Remark 2. When $\varsigma \rightarrow \infty, \eta_{n}=0$, for all $n$, that is, there is no changeover time or service during $\mathrm{WV}$, the model reduces to $\mathrm{GI} / M(n) / 1 / N$ queue with multiple vacations. In absence of $N$-policy, the above results match analytically with the results given by Chao and Rahman [11].

Remark 3. When $\varsigma \rightarrow \infty, \eta_{n}=\eta, \gamma_{n}=\gamma, \mu_{n}=\mu$, for all $n$, that is, there are state independent services and vacations, our model reduces to $\mathrm{GI} / M / 1 / N$ queue with $\mathrm{MWV}$ and results match with Banik et al. [5].

Remark 4. When $\varsigma \rightarrow \infty, \eta_{n} \rightarrow 0$, for all $n$, there is no WV and the model becomes state independent services with vacations. The matching has been done numerically with those of Tian et al. [13].

\section{Computational Algorithm}

To demonstrate the computational aspect for obtaining the prearrival epoch probabilities $P_{n, j}^{-}$at steady state, the following algorithm is developed based on the analysis of Section 3. The computational complexity of the algorithm is $O\left(N^{3}\right)$, where $N$ is the maximum capacity of the system.

Step 1. For $n=0,1, \ldots, N$, calculate $\pi_{n, 0}(0)$ in terms of $\pi_{N, 0}(0)$ as follows:

$$
\begin{array}{ll}
P_{n, 0}(0)=\psi_{n} P_{N, 0}(0), & 0 \leq n \leq N, \\
P_{n, 0}^{*}(\theta)=\zeta_{n, \theta} P_{N, 0}(0), & 1 \leq n \leq N,
\end{array}
$$

where $\psi_{n}$ and $\zeta_{n, \theta}$ are computed below.

(i) From (16) to (17), calculate $\psi_{n}$ as follows:

$$
\begin{gathered}
\psi_{N}=1, \quad \psi_{N-1}=\frac{1-A^{*}\left(\delta_{N}\right)}{A^{*}\left(\delta_{N}\right)}, \\
\psi_{n-1}=\frac{\psi_{n}-\eta_{n+1} \zeta_{n+1, \delta_{n}}}{A^{*}\left(\delta_{n}\right)}, \quad n=N-1, \ldots, 1 .
\end{gathered}
$$

(ii) Calculate $\zeta_{n, \theta}$ from (18) to (19) and from (24) to (25) as given in Algorithm 1.

(iii) Calculate $\zeta_{n, \theta}^{(l)}$ as in Algorithm 2.

Step 2. For $n=N, N-1, \ldots, 1$, calculate $P_{n, 1}(0)$ and $P_{n, 1}^{*}(\theta)$ in terms of $P_{N, 0}(0)$ and $P_{N, 1}(0)$ as follows:

$$
\begin{gathered}
P_{n, 1}(0)=t_{n} P_{N, 0}(0)+d_{n} P_{N, 1}(0), \quad 0 \leq n \leq N, \\
P_{n, 1}^{*}(\theta)=e_{n, \theta} P_{N, 0}(0)+f_{n, \theta} P_{N, 1}(0), \quad 1 \leq n \leq N,
\end{gathered}
$$

where $t_{n}, d_{n}, e_{n, \theta}, f_{n, \theta}$ are given by the following. 


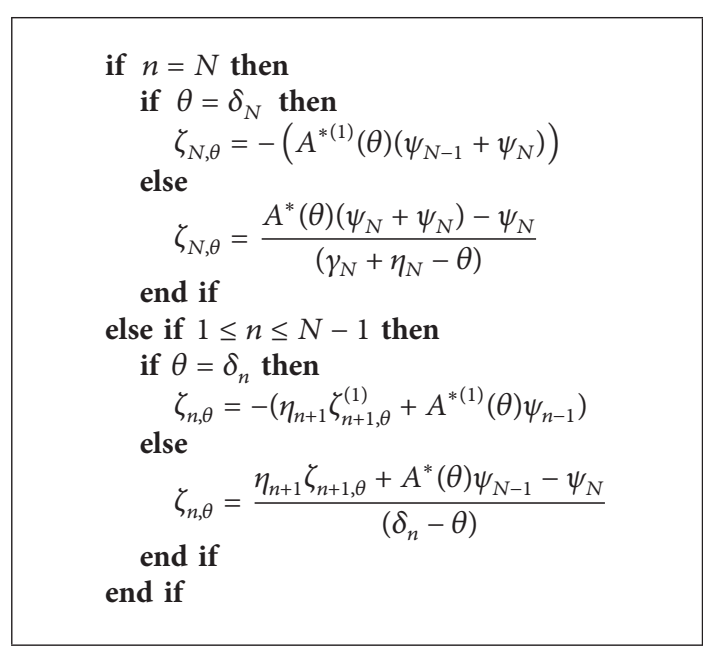

Algorithm 1

(i) From (20) to (21), calculate $t_{n}$ and $d_{n}$ as follows:

$$
\begin{gathered}
t_{N}=0, \quad d_{N}=1, \quad t_{N-1}=-\frac{\gamma_{N} \zeta_{N, \mu_{N}}}{A^{*}\left(\mu_{N}\right)}, \\
d_{N-1}=\frac{1-A^{*}\left(\mu_{N}\right)}{A^{*}\left(\mu_{N}\right)}, \\
t_{n-1}=\frac{t_{n}-\mu_{n+1} e_{n+1, \mu_{n}}-\gamma_{n} \zeta_{n, \mu_{n}}}{A^{*}\left(\mu_{n}\right)}, \\
d_{n-1}=\frac{d_{n}-\mu_{n+1} f_{n+1, \mu_{n}}}{A^{*}\left(\mu_{n}\right)}, \quad 1 \leq n \leq N-1 .
\end{gathered}
$$

(ii) Calculate $e_{n, \theta}$ and $f_{n, \theta}$ from (22) to (27) as shown in Algorithm 3.

(iii) Calculate $e_{n, \theta}^{(l)}$ and $f_{n, \theta}^{(l)}$ as in Algorithm 4.

Step 3. Compute $P_{N, 1}(0)$ in terms of $P_{N, 0}(0)$ as follows:

$$
P_{N, 1}(0)=k P_{N, 0}(0),
$$

where $k=\left(\mu_{1} e_{1, \varsigma}-t_{0}\right) /\left(d_{0}-\mu_{1} f_{1, \varsigma}\right)$.

Step 4. Determine $P_{N, 0}(0)$ from (15) as follows:

$$
P_{N, 0}(0)=\lambda\left[\sum_{n=0}^{N}\left(\psi_{n}+\left(t_{n}+k d_{n}\right)\right)\right]^{-1}
$$

Step 5. The prearrival epoch probabilities $\left(P_{n, j}^{-}\right)$can be evaluated from the rate probabilities $P_{n, j}(0), 0 \leq n \leq N-1, j=0,1$ using (28).

\section{Performance Measures and Numerical Results}

In this section, we discuss some operating characteristics of the queueing system such as average number of customers in

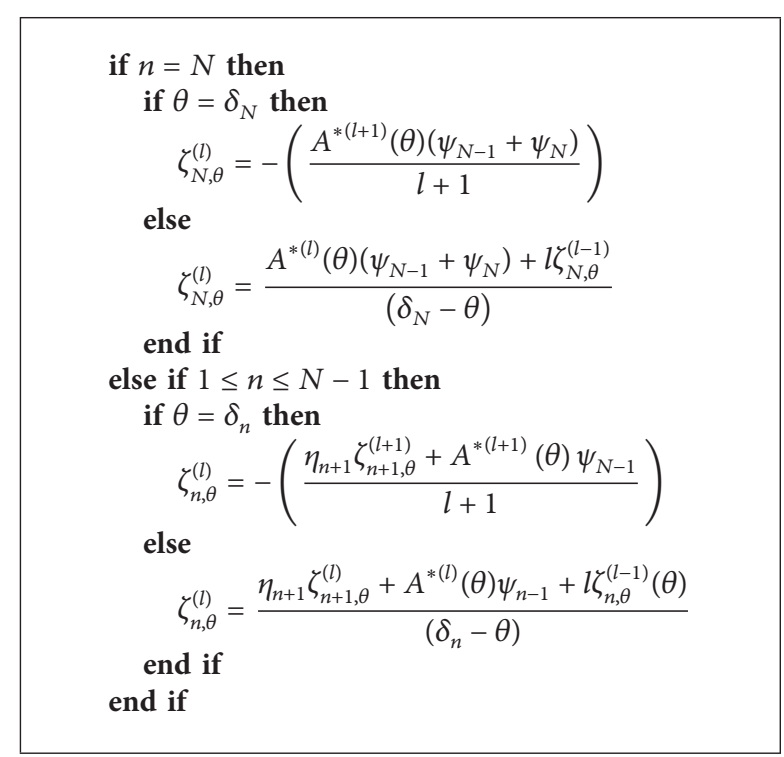

Algorithm 2

the queue $\left(L_{q}\right)$, average number of customers in the system $\left(L_{s}\right)$, and the blocking probability of the server $\left(P_{\text {loss }}\right)$. They are given by

$$
\begin{gathered}
L_{q}=\sum_{n=1}^{N}(n-1) \pi_{n, 0}+\sum_{n=1}^{N}(n-1) \pi_{n, 1} \\
L_{s}=\sum_{n=1}^{N} n \pi_{n, 0}+\sum_{n=1}^{N} n \pi_{n, 1} \\
P_{\text {loss }}=\pi_{N, 0}^{-}+\pi_{N, 1}^{-} .
\end{gathered}
$$

The average waiting time of a customer in the queue (system) $W_{q}\left(W_{s}\right)$ using Little's rule is given by $W_{q}=L_{q} / \widehat{\lambda},\left(W_{s}=\right.$ $\left.L_{s} / \widehat{\lambda}\right)$, where $\hat{\lambda}=\lambda\left(1-P_{\text {loss }}\right)$ is the effective arrival rate.

To validate the computational algorithm, some numerical results are presented in the form of tables and graphs. For computational purpose we have arbitrarily chosen exponential $(M)$, Erlang- $k\left(E_{k}\right)$, deterministic $(D)$ and hyperexponential $\left(\mathrm{HE}_{2}\right)$ interarrival distributions. The capacity of the system is fixed at $N=15$. The traffic intensity is taken as $\rho=$ 0.7 and the various parameters of the model are assumed to be $\varsigma=1.5, \mu_{n}=0.5 n, \gamma_{n}=0.15 n$, and $\eta_{n}=0.3 n(1 \leq n \leq N)$ with means $\mu=4.0, \gamma=1.2, \eta=2.4$, respectively. For $\mathrm{HE}_{2}$ distribution, we have taken $\lambda_{1}=1.88235, \lambda_{2}=3.2, \sigma_{1}=0.4$, and $\sigma_{2}=0.6$.

Table 1 presents the sensitivity analysis for various models like state dependent and MWV with changeover time (state + $\mathrm{MWV}+$ chover), state dependent and MWV (state + MWV), and constant with MWV (constant + MWV) models. The results are obtained by considering exponential interarrival time distribution. Note that for exponential distribution, the prearrival and arbitrary epoch probabilities are the same due to the memoryless property. So, we have presented only arbitrary epoch probabilities in the table. Moreover, as $\varsigma \rightarrow$ $\infty$, we obtain models with state dependent and constant 
TABLE 1: Sensitivity analysis of $M / M(n) / 1 / 5$ system $(\rho=0.333, \mu=1.5, \eta=0.45, \gamma=0.9)$.

\begin{tabular}{|c|c|c|c|c|c|c|}
\hline \multirow{2}{*}{$n$} & \multicolumn{2}{|c|}{ State + MWV + chover } & \multicolumn{2}{|c|}{ State + MWV } & \multicolumn{2}{|c|}{ Constant + MWV } \\
\hline & $P_{n, 0}$ & $P_{n, 1}$ & $P_{n, 0}$ & $P_{n, 1}$ & $P_{n, 0}$ & $P_{n, 1}$ \\
\hline 0 & 0.250666 & 0.046661 & 0.287054 & - & 0.412858 & - \\
\hline 1 & 0.184471 & 0.186645 & 0.211250 & 0.160304 & 0.141381 & 0.119402 \\
\hline 2 & 0.083190 & 0.135643 & 0.095267 & 0.128617 & 0.048455 & 0.107192 \\
\hline 3 & 0.026924 & 0.056790 & 0.030832 & 0.056128 & 0.016691 & 0.073488 \\
\hline 4 & 0.006845 & 0.016821 & 0.007838 & 0.017037 & 0.005926 & 0.045490 \\
\hline 5 & 0.001521 & 0.003820 & 0.001741 & 0.003929 & 0.002469 & 0.026644 \\
\hline Sum & 0.553619 & 0.446381 & 0.633984 & 0.366016 & 0.627782 & 0.372218 \\
\hline$L_{q}$ & 0.478630 & & 0.495121 & & 0.606714 & \\
\hline$P_{\text {loss }}$ & 0.005341 & & 0.005671 & & 0.029113 & \\
\hline$W_{q}$ & 0.962401 & & 0.995890 & & 1.249820 & \\
\hline
\end{tabular}

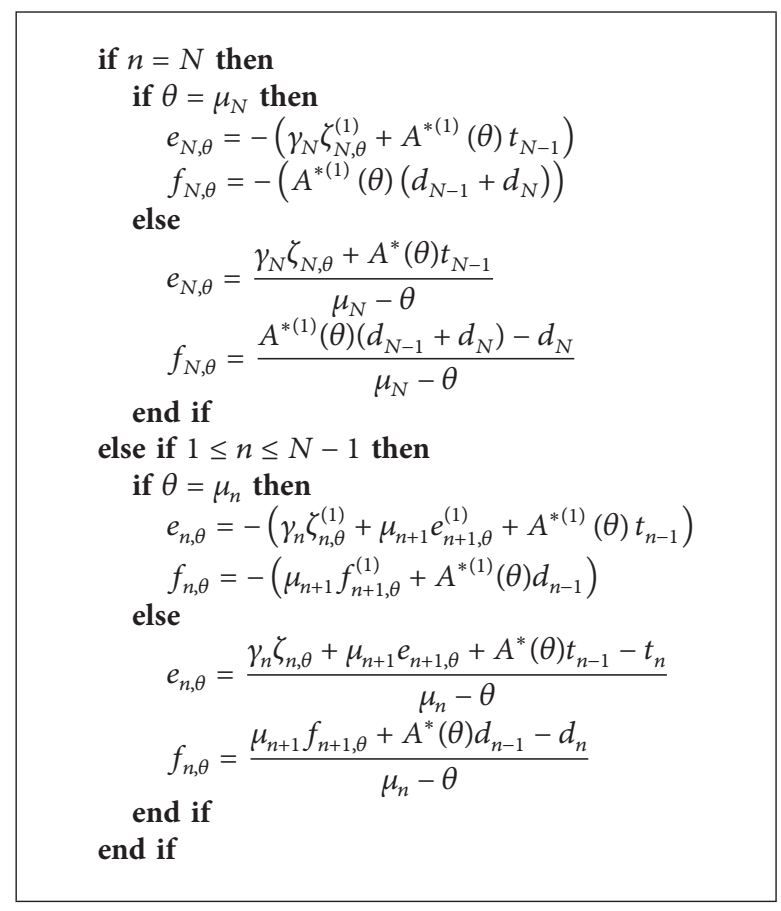

Algorithm 3

service rates. Further, the performance measures $P_{\text {loss }}, L_{q}, W_{q}$ are lower in state $+\mathrm{MWV}+$ chover queue. Finally, the model with changeover time gives better results and justifies our analysis.

Figure 1 compares the effect of arrival rate $\lambda$ on the expected queue length $L_{s}$ among the models: (i) state + MWV + chover, (ii) constant + MWV + chover, (iii) state + MWV, and (iv) constant + MWV for the deterministic interarrival time distribution. It is clear that $L_{s}$ increases as arrival rate increases in all the above models. Further, among the four models considered, the state dependent model with changeover time performs best.

Figure 2 illustrates the effect of mean service rate during WV $(\eta)$ on the average waiting time in the system $\left(W_{s}\right)$ for different mean vacation rates $\gamma=0.3,0.45,0.6$ (by

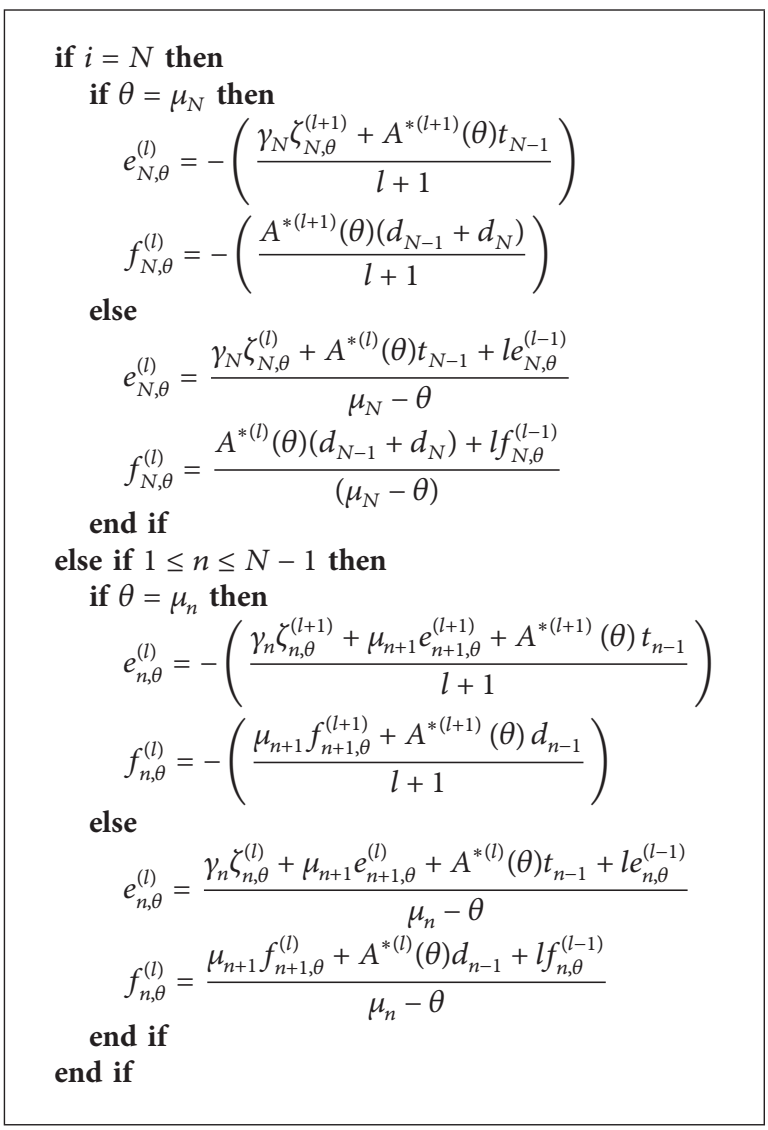

Algorithm 4

considering $\gamma_{n}=0.1 n, \gamma_{n}=0.15, \gamma_{n}=0.2$ ) for exponential interarrival time. Considering the parameters by $N=5, \mu_{n}=$ $0.5 n$ (1.5) $\forall n$, we observe that $W_{s}$ decreases as $\eta$ increases. Further, as the mean vacation rate $\gamma$ increases, the $W_{s}$ decreases and meets at a point $\eta=\mu=1.5$. Therefore, the WV queue utilizes the idle time effectively when $\eta \leq \mu$.

Figure 3 depicts the effect of arrival rate $\lambda$ on average system length $L_{q}$ with different values of $\varsigma$ for $E_{3}$ distribution. It may be observed that $L_{q}$ increases with arrival rate. Further, increase of $\varsigma$ also results in the increase of queue lengths. 


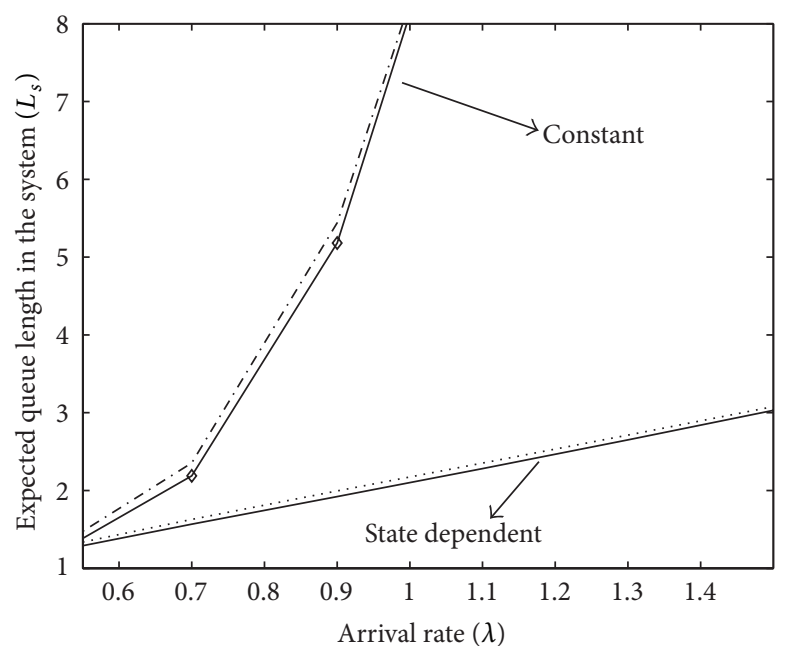

$\begin{array}{lll}\text { - } D / M(n) / 1 / 15 / \mathrm{MWV}+\text { chover } & \cdots \cdots & D / M(n) / 1 / 15 / \mathrm{MWV} \\ \text {-.- } D / M / 1 / 15 / \mathrm{MWV}+\text { chover } & \rightarrow & D / M / 1 / 15 / \mathrm{MWV}\end{array}$

Figure 1: Effect of $\lambda$ on $L_{s}$.

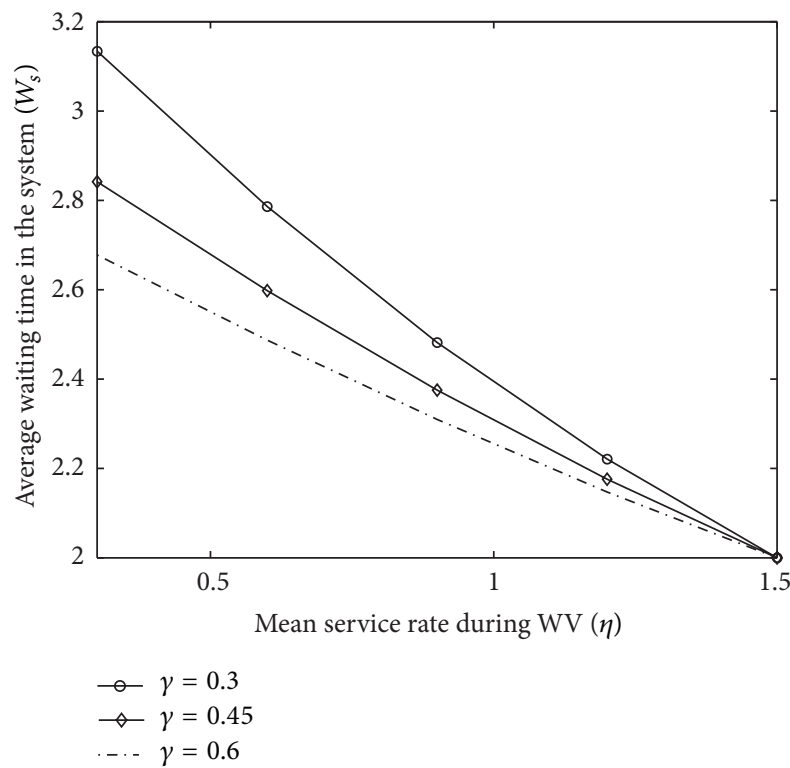

FIGURE 2: Impact of $\eta$ on $W_{s}$.

The effect of buffer size $N$ on blocking probability $P_{\text {loss }}$ for various interarrival time distributions is shown in Figure 4. We observe that as the buffer size increases the $P_{\text {loss }}$ decreases. Furthermore, $P_{\text {loss }}$ is the highest in case of $\mathrm{HE}_{2}$ distribution, whereas deterministic distribution yields the lowest.

\section{Conclusions}

This paper presents a single server state dependent queue with MWV and changeover time. The interarrival time of customer arrival is arbitrarily distributed, while the service rates during regular busy period and during $\mathrm{WV}$ and vacation rate are exponentially distributed. A recursive method has

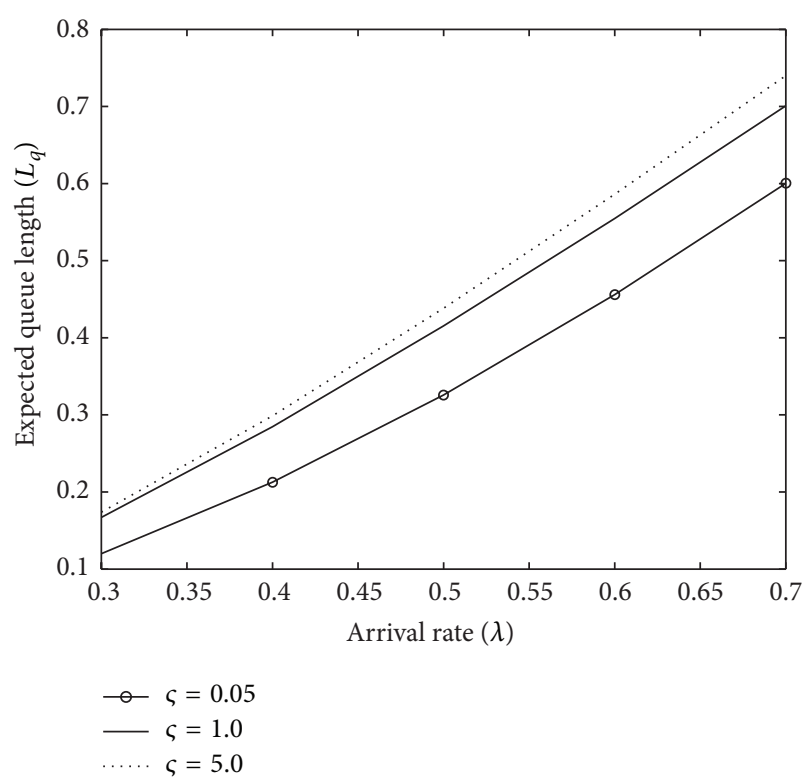

Figure 3: Effect of $\lambda$ on $L_{q}$.

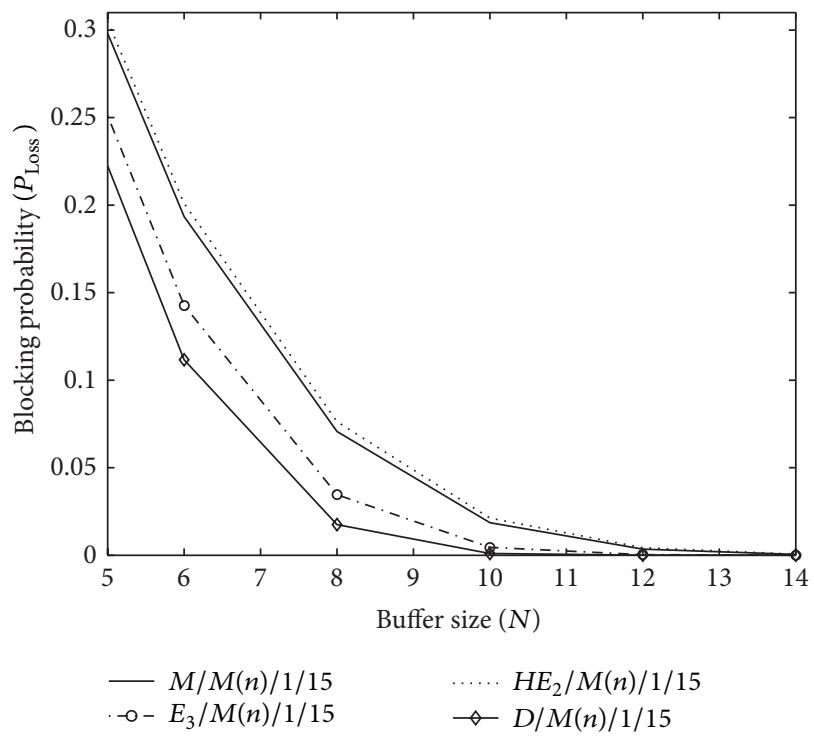

Figure 4: Impact of $N$ on $P_{\text {loss }}$.

been developed to obtain the steady state queue length distributions at prearrival and arbitrary epochs. Numerical results have also been discussed. The changeover period concepts can be added to analyze more complex models like $\mathrm{GI} / \mathrm{Geo} / 1 / N$ and $\mathrm{GI} / G / 1 / N$ queues with various services and vacation policies.

\section{Conflict of Interests}

The authors declare that there is no conflict of interests regarding the publication of this paper. 


\section{References}

[1] B. T. Doshi, "Queueing systems with vacations-a survey," Queueing Systems, vol. 1, no. 1, pp. 29-66, 1986.

[2] N. Tian and Z. G. Zhang, Vacation Queueing Models: Theory and Applications, Springer, New York, NY, USA, 2006.

[3] L. D. Servi and S. G. Finn, "The $M / M / 1$ queues with working vacations $(M / M / 1 / W V)$," Performance Evaluation, vol. 50, no. 1, pp. 41-52, 2002.

[4] Y. Baba, "Analysis of a GI/M/1 queue with multiple working vacations," Operations Research Letters, vol. 33, no. 2, pp. 201209, 2005.

[5] A. D. Banik, U. C. Gupta, and S. S. Pathak, "On the GI/M/1/N queue with multiple working vacations-analytic analysis and computation," Applied Mathematical Modelling, vol. 31, no. 9, pp. 1701-1710, 2007.

[6] M. Zhang and Z. Hou, "Steady state analysis of the GI/M/1/N queue with a variant of multiple working vacations," Computers and Industrial Engineering, vol. 61, no. 4, pp. 1296-1301, 2011.

[7] H. Li and Y. Zhu, "Analysis of $M / G / 1$ queues with delayed vacations and exhaustive service discipline," European Journal of Operational Research, vol. 92, no. 1, pp. 125-134, 1996.

[8] H. H. Dong and I. C. Doo, "G/M/1 queues with delayed vacations," Korean Journal of Computer \& Applied Mathematics, vol. 5, pp. 1-11, 1998.

[9] M. Jain and P. Singh, "State dependent bulk service queue with delayed vacations," JKAU Engineering Sciences, vol. 16, pp. 3-14, 2005.

[10] P. Vijaya Laxmi and D. Selshi, "Performance analysis of renewal input $(a, b, c)$ policy with multiple working vacation and changeover times," Journal of Industrial and Management Optimization, vol. 10, pp. 839-857, 2013.

[11] X. Chao and A. Rahman, "Analysis and computational algorithm for queues with state-dependent vacations I: G/M(n)/1/K," Journal of Systems Science and Complexity, vol. 19, no. 1, pp. 36-53, 2006.

[12] V. Goswami, P. Vijaya Laxmi, and K. Jyothsna, "Analysis of $G I / M(n) / 1 / N$ queue with state dependent multiple working vacations," Opsearch, vol. 50, pp. 106-124, 2013.

[13] N. Tian, D. Zhang, and C. Cao, "The GI/M/1 queue with exponential vacations," Queueing Systems, vol. 5, no. 4, pp. 331344, 1989. 


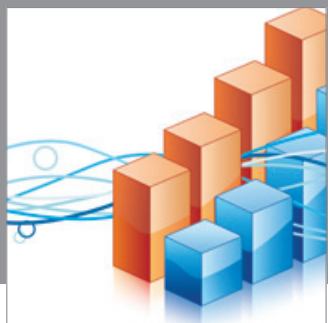

Advances in

Operations Research

mansans

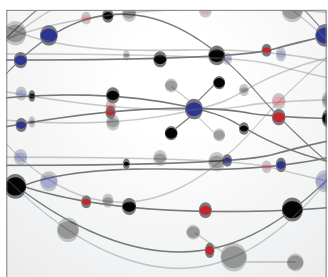

The Scientific World Journal
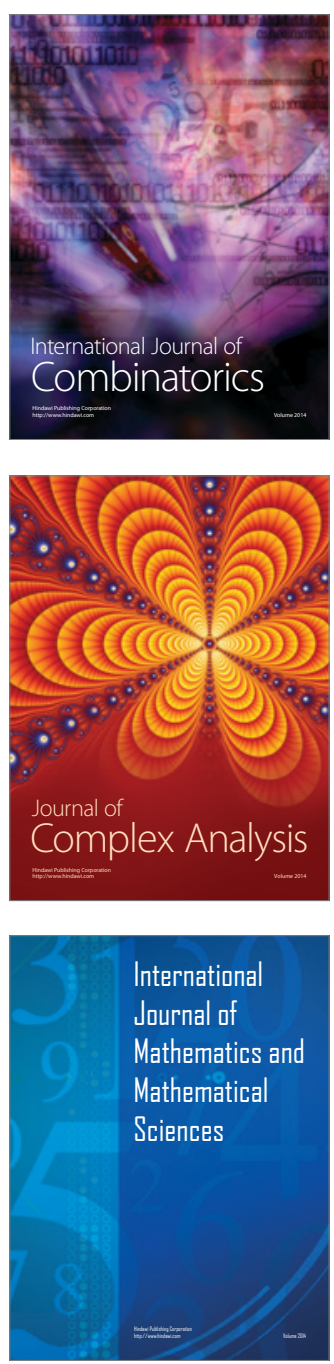
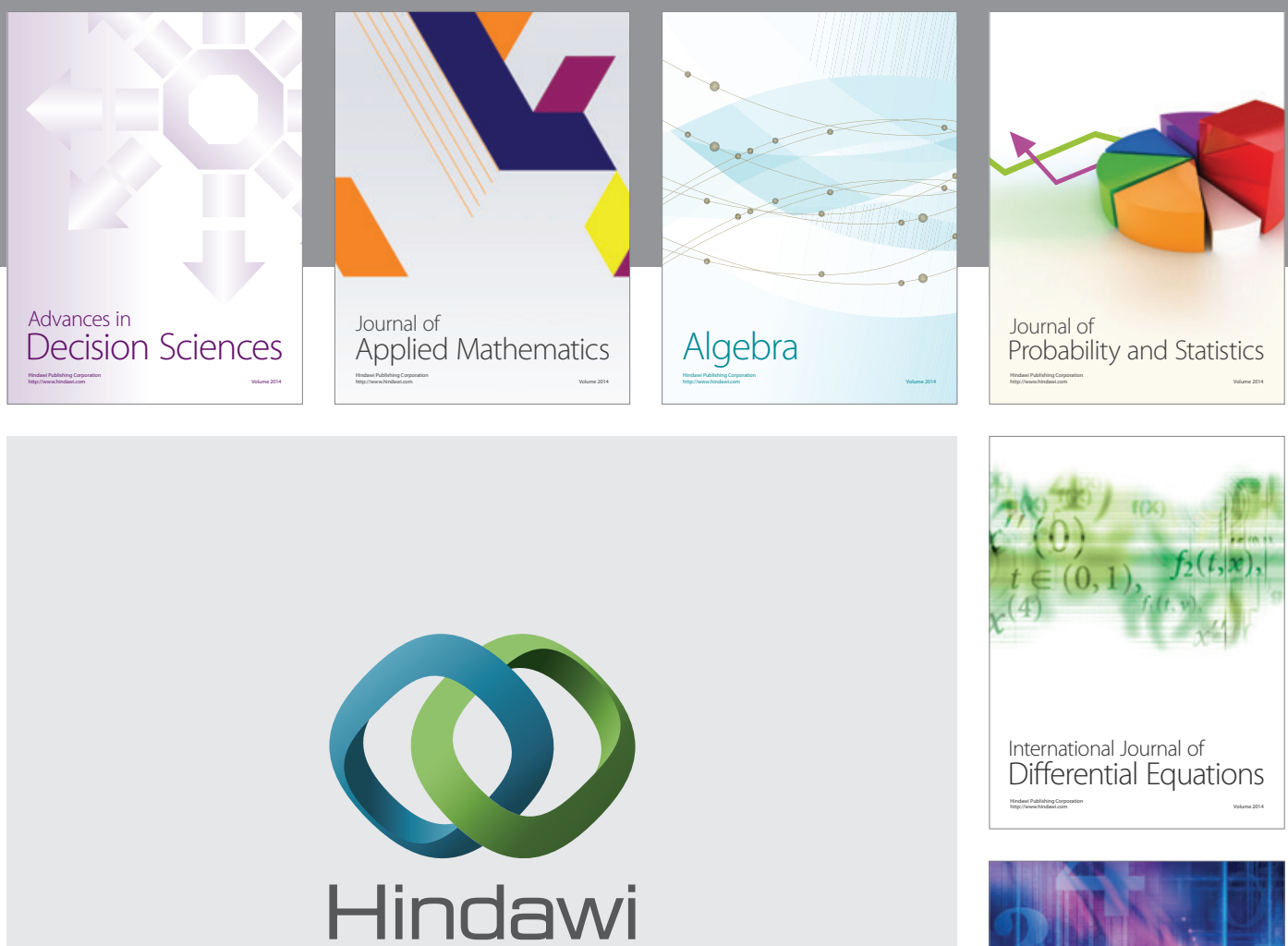

Submit your manuscripts at http://www.hindawi.com
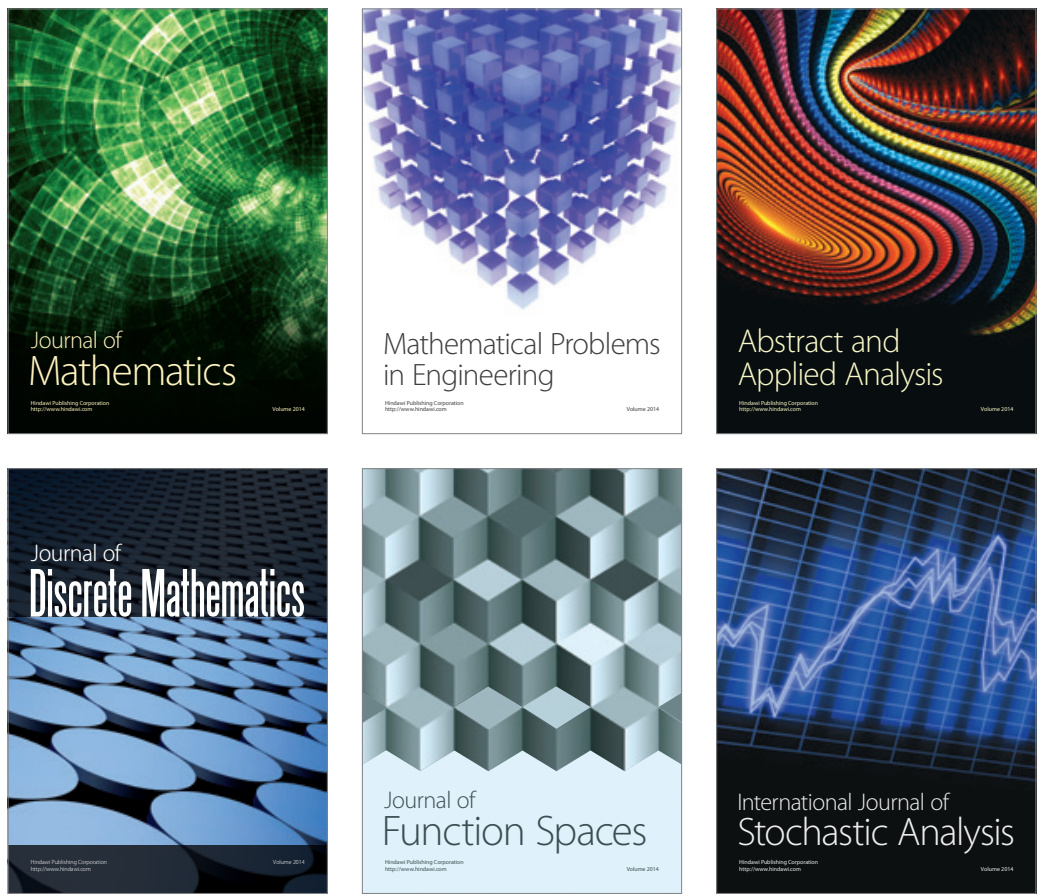

Journal of

Function Spaces

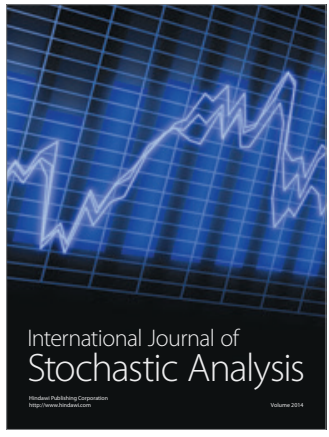

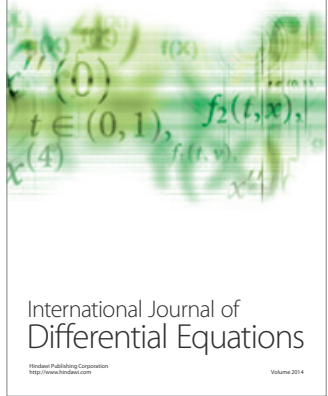
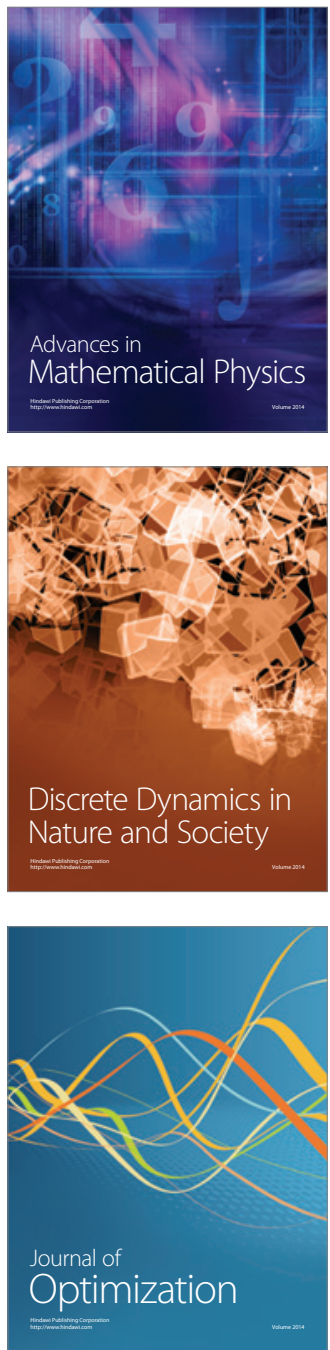